\section{Amantidine in neuroleptic malignant syndrome}

Sir,

We read with interest the case report by Woo et al.' regarding successful treatment of an unusually long case of neuroleptic malignant syndrome (NMS) by amantidine.

Though we agree with the diagnosis of NMS, we feel that some useful information about the case has not been provided by the authors. Despite the fact that the diagnosis was made prospectively the authors have failed to give detailed description. Since most of the literature on NMS is limited to case report material, for further understanding of this condition it is highly desirable that maximum information about the case should be made available. In fact it has been commented earlier by different reviewers on the subject that most of the case reports have provided information about only a limited number of variables choosing to be silent on variables which were not deranged..$^{2,3}$ Even a statement about normality of a particular variable may be useful for further research.

The authors in this case have made a blanket statement about absence of any haematological, biochemical or microbiological abnormalities in blood or cerebrospinal fluid. It would have been better if the authors had mentioned specifically about serum CPK levels, total leucocyte count and serum levels of liver enzymes. Even if the aforementioned parameters were normal a specific statement would have been useful as these parameters are more often deranged in NMS. Surprisingly, despite such a long duration of NMS in this case, the authors did not plan a muscle biopsy though it is not essential for making the diagnosis. ${ }^{2}$

What is unusual in this case is the abnormally long duration of NMS which persisted for 3 weeks after cessation of neuroleptics. Such a duration has been seen more commonly with depot neuroleptics rather than oral owing to the greatly extended half-life of depot neuroleptics. ${ }^{2}$

Relapse after a further 17 days of amantidine treatment is another unusual feature hitherto not mentioned in the literature. The clinical course suggests that the neuroleptic or its active break-down products persisted in the circulation for more than 5 weeks. We wonder whether this had something to do with the metabolic characteristics of this patient. Absorption characteristics and first-pass metabolism have been known to cause wide inter-individual variations in plasma levels of neuroleptics. Racial and genetic differences in the metabolism of neuroleptics like ethanol may explain such an unusually long course.

The dogmatic statement by the authors about this being the second case successfully treated with amantidine is really not true. There are at least four case reports prior to this. ${ }^{4.5,6,7}$ Recently there was another case report about successful treatment of NMS with amantidine. ${ }^{8}$ The reference to Buze and Baxter should have read Guze and Baxter.'

Adityan Jee, Department of Psychological Medicine, Faculty of Medicine, University of Malaya, 59100, Kuala Lumpur, Malaysia.

\section{References}

1. Woo, J., Teoh, R. \& Vallance-Owen, J. Neuroleptic malignant syndrome successfully treated with amantidine. Postgrad Med J 1986, 62: 809-810.

2. Levenson, J.L. Neuroleptic malignant syndrome. Am J Psychiatry 1985, 142: 1137-1145.

3. Shalev, A. \& Munitz, H. The neuroleptic malignant syndrome: agent and host interaction. Acta Psychiatr Scand 1986, 73: 337-347.

4. McCarron, M.M., Boettger, M.L. \& Peck, J.J. A case of neuroleptic malignant syndrome successfully treated with amantidine. J Clin Psychiatry 1982, 43: 381-382.

5. Gangadhar, B.N., Desai, N.G. \& Channabasavanna, S.M. Amantidine in the neuroleptic malignant syndrome (letter). J Clin Psychiatry 1984, 45: 526.

6. Amdurski, S., Radwan, M., Levi, A. \& Elizur, A. A therapeutic trial of amantidine in haloperidol-induced malignant neuroleptic syndrome. Curr Ther Res 1983, 33: 225-229.

7. Rojtman, M., Apter, E., Lahav, S. \& Tiano, S. Amantidine in malignant neuroleptic syndrome (English Translation). Harefuah 1981, 100: 333.

8. Hamburg, P., Weilburg, J.B., Cassem, N.H., Cohen, L. \& Brown, S. Relapse of neuroleptic malignant syndrome with early discontinuation of amantidine therapy: Compr Psychiatry 1986, 27: 272-275.

9. Guze, B.H. \& Baxter, L.R. Neuroleptic malignant syndrome. $N$ Engl J Med 1985, 313: 163-166.

This letter has been shown to $\mathrm{Dr}$ Woo who replies:-

Sir,

We thank Dr Jee for the points raised in his letter.

It is precisely because of the unusual features he mentioned that we were prompted to report the case. These being, the long duration of the NMS which persisted for 3 weeks after stopping neuroleptics, and relapse after completion of a 17 day course of amantidine.

The blanket statement that no laboratory abnormalities were found was made to emphasize the absence of other causes of fever. The limitation of space precluded a full description of normal results: these included a complete blood picture, sedimentation rate, renal and liver function tests, blood glucose, blood and cerebrospinal (CSF) serology for viral antigens including Japanese B encephalitis, blood for ANF and RA titre, LE cell screen; CSF cell count, protein, glucose, smear for acid fast bacilli and cryptococcus; blood, CSF, urine, sputum and stool cultures for ordinary bacteria and mycobacteria. The serum GPT was transiently elevated to $76 \mathrm{IU} / \mathrm{l}$ (upper limit: $60 \mathrm{IU} / \mathrm{l}$ ) 5 days after the onset of fever. As the diagnosis was not made at presentation, the serum creatine kinase was not done. Though a muscle biopsy would have been of interest, it could not be justified on diagnostic grounds.

The review mentioned' was published after submission of 
our case report and quoted two additional cases of the NMS which responded to amantidine.

\section{Jean Woo \\ Robert Teoh \\ John Vallance-Owen Department of Medicine, Prince of Wales Hospital, Chinese University of Hong Kong, Shatin, NT, Hong Kong.}

\section{References}

1. Shalev, A. \& Munitz, H. The neuroleptic malignant syndrome: agent and host interaction. Acta Psychiatr Scand 1986, 73: 337-347.

\section{Crohn's colitis and sarcoidosis}

Sir,

The similarities between Crohn's disease and sarcoidosis were emphasized in the case reported by Dr McCormick and his colleagues.' Cases of Crohn's disease sometimes show positive Kveim tests, ${ }^{2}$ and the disorder is, at times, indistinguishable from ulcerative colitis. ${ }^{3}$ BCG inoculation may also conceivably account for the apparent increase in its incidence. ${ }^{4}$ The difference between Crohn's disease and sarcoidosis is slight, and it is probably due to slightly different transmissible agents. Sarcoidosis is possibly due to an attenuated human mycobacterium, while Crohn's disease may be caused by a bovine and attenuated mycobacterium.

\section{Gerald MacGregor Percy Place, \\ 31 Epsom Road, Guildford, GUJ $3 L A, U K$.}

\section{References}

1. McCormick, P.A., O’Donoghe, D.P. \& Fitzgerald, M.X. Crohn's colitis and sarcoidosis. Postgrad Med J 1986, 62: 951-953.

2. Karlish, A.J., Cox, E.V., Hampson, F. \& Hemsted, E.H. Kveim test in Crohn's disease. Lancet 1972, i: 438-439.

3. MacGregor, G.A. Three reasons for ulcerative colitis. Lancet 1973 , i; 825-826.

4. MacGregor, G.A. BCG: Bad news from India. Lancet 1980 , i: 318 .

\section{Fansidar - a treatment for AIDS-related pneumocystis?}

\section{Sir,}

The standard treatment of acquired immune deficiency syndrome (AIDS) associated Pneumocystis carinii pneumonia (PCP) is high dose co-trimoxazole (trimethoprim and sulphamethoxazole). ${ }^{1}$ This drug results in a severe hypersensitivity reaction in more than $50 \%$ of patients. Pentamidine is an established alternative, but poor patient tolerance is common and the intramuscular route is contraindicated in thrombocytopenia. Low dose Fansidar (one tablet weekly of pyrimethamine $25 \mathrm{mg}$, sulfadoxine $500 \mathrm{mg}$ ) has been used as an alternative agent to co-trimoxazole in prophylaxis against recurrence of AIDS-related PCP. ${ }^{2}$ We describe here the apparently successful use of high dose Fansidar (three tablets weekly) in the treatment of PCP in three patients intolerant of co-trimoxazole.

Comparative details of the three patients are given in Table I. All patients were human immunodeficiency virus (HIV) antibody positive and PCP was confirmed using trans-bronchial biopsy or lavage. All patients were treated initially with co-trimoxazole. In each case, a hypersensitivity rash appeared between 5 and 12 days following introduction of the drug. Patient 1 also received trimethoprim, which again resulted in a hypersensitivity rash. None of the patients received post-PCP prophylaxis or folic acid supplements.

None of our patients treated with Fansidar has suffered a relapse of their PCP over a period of at least 3 months in spite of not receiving prophylaxis. This is surprising in a disease exhibiting a median survival time of 9 months but may partly reflect the long half-life of Fansidar (130 hours).

Fansidar is not without side effects. These include elevation of liver enzymes and occasionally hepatitis; the former we saw in patient 1 , who had the longest period of treatment. Marrow suppression may also occur, resulting in agranulocytosis, perhaps related to changes in folic acid metabolism. Patients 1 and 3 had falls in cell counts. Close monitoring of chemistry and haematological parameters is recommended.

Cutaneous adverse reactions, including Stevens-Johnson syndrome, have also been reported. ${ }^{3}$ However, in limited and cautious use, $75 \%$ of patients reacting to co-trimoxazole could tolerate Fansidar. ${ }^{4}$ This observation and the reaction of our patient 1 to trimethoprim suggest that the constituent sulphonamides are not always the problem and that some AIDS patients may be specifically intolerant of trimethoprim.

It can be argued that the clinical improvements seen in our patients were a delayed response to prior therapy. However, the symptomatic improvement (and radiological clearing in patients 1 and 3 ) seen only after starting Fansidar may be significant. Certainly a controlled comparative trial of cotrimoxazole and Fansidar is warranted. Until then we consider that Fansidar is worth trying in the treatment of lifethreatening PCP, particularly in patients sensitive to or intolerant of co-trimoxazole and pentamidine.

\section{References}

1. Murray, J.F., Felton, C.P., Garay, S.M. et al. Pulmonary complications of the acquired immunodeficiency syndrome. $N$ Engl J Med 1984, 310: 1682-1688

2. Gottleib, M.S., Knight, S., Mitsuyasu, R. et al. Prophylaxis of Pneumocystis carinii infection in AIDS with pyrimethamine-sulfadoxine. Lancet 1984, ii: 398-399

3. Navin, T.R., Miller, K.D., Satriale, R.F. \& Lobel, H.O. Adverse reactions associated with pyrimethamine-sulfadoxine prophylaxis for pneumocystis carinii infections in AIDS. Lancet 1985, i: 1332 